\title{
Mapping Cyberspace
}

\author{
Elizabeth A. McDaniel and Michael J. Piller \\ National Defense University iCollege, Washington, DC, USA \\ Mcdaniele@ndu.edu pillerm@ndu.edu
}

\begin{abstract}
This paper reports on a project designed to advance understanding of cyberspace, a new and evolving global domain with particular value for national security. Faculty members in a government graduate school whose mission is to develop leaders who can leverage information and information technology for national security participated in the project to map cyberspace. Using a dynamic mind mapping tool called The Brain, the faculty created an original map of cyberspace to facilitate understanding and communication about its complexity and scope.
\end{abstract}

Keywords: cyberspace, concept mapping, knowledge mapping.

\section{Introduction}

According to Wikipedia:

Now ubiquitous, in current usage the term "cyberspace" stands for the global network of interdependent information technology infrastructures, telecommunications networks and computer processing systems. As a social experience, individuals can interact, exchange ideas, share information, provide social support, conduct business, direct actions, create artistic media, play games, engage in political discussion, and so on, using this global network. The term has become a conventional means to describe anything associated with the Internet and the diverse Internet culture. The United States government recognizes the interconnected information technology and the interdependent network of information technology infrastructures operating across this medium as part of the US National Critical Infrastructure (National Strategy to Secure Cyberspace, 2003) Retrieved December 28, 2011, from http://en.wikipedia.org/wiki/Cyberspace.

Because of the potential power and impact of cyberspace, the U.S. Department of Defense has identified cyberspace as a war-fighting domain. According to the National Military Strategy for Cyberspace Operations (NMS-CO), cyberspace is "a domain characterized by the use of electronics and the electromagnetic spectrum to store, modify, and exchange data via networked sys-

Material published as part of this publication, either on-line or in print, is copyrighted by the Informing Science Institute. Permission to make digital or paper copy of part or all of these works for personal or classroom use is granted without fee provided that the copies are not made or distributed for profit or commercial advantage AND that copies 1) bear this notice in full and 2) give the full citation on the first page. It is permissible to abstract these works so long as credit is given. To copy in all other cases or to republish or to post on a server or to redistribute to lists requires specific permission and payment of a fee. Contact Publisher@InformingScience.org to request redistribution permission. tems and associated physical infrastructure" (December, 2006). This definition establishes cyberspace as a war-fighting domain with characteristics similar to the traditional war-fighting domains of land, sea, air, and space. The definition makes it a physical domain by establishing physical boundaries to the domain in the form of the electromagnetic spectrum (EMS). It encompasses all things of, relating to, or within the EMS, in- 
cluding all cyberspace-related activities, infrastructures, people, and telecommunications and information systems that comprises "electronics" as the means or tools to conduct cyber warfare.

"Cyber" has become an adjective used to modify many words in our current culture, such as cyber security, cyber law, and cyber space. Cyber topics populate almost every issue of daily national newspapers because of their significance, ubiquity, and potential impact on our lives. $\mathrm{Cy}-$ ber the adjective and cyber the reality have become integral to our organizations and our lives in small steps and huge leaps. But despite the fact that we are living it, we have not created metaphors or mental models for understanding cyberspace, perhaps because it is virtual, ephemeral, boundary-less, exponential in its growth, ubiquitous and global, and personal as well as organizational and institutional. Understanding the scope, complexity, and interconnectedness of cyberspace can facilitate our ability to leverage it for our purposes and to manage its impact on our lives.

\section{Mapping}

Mapping is an approach for externalizing concepts to make them meaningful, understood, and manageable. Now used in various fields for different purposes, mapping is sometimes called concept mapping, mind mapping, argument mapping, and knowledge mapping. According to Peter Senge, mental models are deeply ingrained assumptions, generalizations, or images that influence how we understand the world and how we take action (1990). They exist in the mind of the individual. Concepts maps are the graphical representation of a mental model.

Concept maps are graphical diagrams that can be used to organize knowledge in meaningful ways (Novak \& Gowin, 1984), and are generally attributed to Novak's early work examining children's understanding of scientific knowledge (1984) that resulted in the notion of graphical concept mapping. They facilitate deep understanding of learning material because learners link new knowledge to previously constructed concepts and propositions (Ausubel, 1963). Concepts are generally represented as nodes or circles, and relationships among concepts are illustrated by connecting lines. Clarifying words or symbols are typically associated with linking lines to explain the specific relationships among the concepts. Although concept maps are generally represented hierarchically (Novak, 1998), with the most general concepts at the top and more specific concepts arranged below, variations also take the form of chain, spider, and network formats (Ruiz-Primo \& Shavelson, 1996). Safayeni, Derbentseva, and Canas (2003) suggest that a cyclic structure more clearly illustrates dynamic relationships and stimulates systems thinking.

Concept mapping, originally designed to enhance learning and memory, is an approach to knowledge structure externalization or external cognition that helps learners initially reconstruct their concepts and consider ways of visually representing their conceptual structures (Kao \& KuanChien Sun, 2010). Mapping facilitates understanding of complex relationships, promotes deep and not surface learning, and requires more active engagement which leads to greater learning (Davies, 2010). Concept mapping is a mindtool that provides learners with the necessary scaffolding to make sense of a complex context; other types of mindtools are databases, spread sheets, and expert systems. Originally designed for paper and pencil, today's concept maps can be developed using computer-based tools or learning environments that serve as extensions of the mind (Jonassen, Carr, \& Yueh, 1998) to assist learners in representing knowledge and learning. Used extensively as a learning tool, concept mapping combined with critical reflection and action learning exercises can help students build better awareness of their emerging mental models and biases and take steps to address knowledge gaps (McLaren, Vuong, \& Grant, 2007). By linking words and images in an intuitive way, mind maps attempt to capture complex abstractions in ways that reflect the organic and free-flowing natural way the mind works (Buzan, 1991). 
In addition to its benefits for learning and memory, mapping is also a powerful tool for knowledge management and making sense in environments characterized by information overload and complexity. According to Wexler, knowledge maps determine what is relevant or organizationally important, and are problem-centered (2001). Mapping enables organizations to bring together their information for making decisions, to consider major and minor topics or issues and their relationships to each other, and to present arguments in clear formats. Dynamic mapping tools foster collaboration that allows participants to add ideas, modify relationships, and enhance the clarity and power of complex ideas.

Advances in information and communication technology, in the form of easy-to-use software applications for personal electronic devices, facilitate visualization in general and mapping in particular for many purposes and fields. Information visualization promotes insight for the purposes of discovery, decision-making, and explanation (Card, Mackinlay, \& Shneiderman, 1999). In the most general sense, information visualization techniques are used to decrease the demands upon working memory, that memory system that is employed during the immediate storage and processing of information (Baddeley, 1986). The goal of information visualization techniques is to offer a computational offloading of working memory processing. In this case computational effort is "offloaded" onto the visualization software, freeing information processing capabilities of the viewer (Navarro-Pietro, Scaife, \& Rogers, 1999).

\section{Process}

A government-sponsored graduate school whose mission is to develop cyber leaders employs fifty faculty members who design and offer courses, deliver presentations, and write articles on various aspects of cyberspace. In the fall of 2011, prompted by a request by a stakeholder organization for a seminar of the landscape of cyberspace, the faculty embarked on the daunting task of mapping cyberspace as a framework for the big ideas and interconnected elements of this new domain.

Faculty members, experts in various aspects of information and information technology, assurance, leadership and management, design and deliver innovative interdisciplinary courses to U.S. government mid- to senior-level employees. Their courses constitute the curricula to prepare chief information officers, chief information security officers, chief technology officers, enterprise architects, information operators, and IT project and program managers.

Twenty-five faculty members participated in the project by contributing at least one hour during fifteen hours of group mapping sessions, and some faculty members returned for a second hour of mapping. Subsequently, selected faculty members, either who were unable to attend the group sessions or who have particular expertise and/or documents, contributed to the map in one-on-one sessions with the project leader.

In this project The Brain software (webbrain.com) was used because it offers dynamic mind mapping capabilities that link non-linear information into networks of non-hierarchical logical associations in ways that mimic the human brain. The Brain is designed to help map creators visualize concepts and ideas, called thoughts, and link them into multiple categories and logical associations. Instead of organizing information into linear files, The Brain operates on associations and captures connections and relationships. Two important characteristics of the tools are its ability to load information dynamically and its infinite scalability. By using a method similar to the hyperbolic tree graph drawing method first introduced by Lamping, Rao, and Pirolli (1995), nodes in focus are placed in the center and are given more room, while out-of-focus nodes are compressed in the background. Although not specifically following hyperbolic geometry, the Brain software uses a graphical layout of topics connected by lines that radiate out from a central topic. With this capability, any thought can dynamically become the central focus in context. 
The big picture, the details, associations and connections can be drawn and dynamically displayed.

\section{Assumptions}

1. Maps are human creations intended to guide users through a complex and/or changing knowledge field (Wexler, 2001).

2. Maps allow users to locate themselves in the context of a larger landscape and relationships to other elements on the map. The mapping tool provides context for each concept and its relationships to other concepts.

3. Multiple maps of cyberspace are possible; there is no one way to map cyberspace, nor one best tool. The map is not the solution or the territory; it directs us to information and facilitates broader understanding.

4. The contents of knowledge maps alter through use, reuse, and experimentation (Wexler, 2001). Initial efforts to map cyberspace will be primitive because cyberspace is evolving rapidly and so much is unknown. The cyberspace map will develop iteratively through ongoing dialog.

5. Faculty members are experts in various aspects of cyberspace, such as security, war, governance, technologies, law, and leadership. One's view of cyberspace is grounded in one's perspective as an expert in a specialty area.

6. Individual contributors to the mapping project have the power to edit the latest draft.

7. A cyberspace map might identify concepts that are not taught in the current curriculum, and prompt the development of new courses.

\section{Observations}

1. The mapping sessions prompted rich conversation and sharing of ideas among participants. According to Wexler, effective map makers facilitate learning, instill curiosity and reward it but also encourage self-correcting action, highlight joint perspectives as well as potential differences, permit exploration of possibilities and rehearsal of options and new relationships, provide clarity and absorb ambiguity (2001).

2. Faculty members varied in their responses to the process. Some experts enthusiastically contributed their ideas and found the evolving map to be interesting and worthwhile. Others expressed confusion about the goal of the project and the value of the final outcome. Some did not like the tool selected for mapping.

3. People, processes, and technology, the lenses through which the faculty tend to analyze topics and issues, are involved in many aspects of cyberspace. But they decided not to address people, processes, and technology specifically for each topic. For example, while technologies are foundational to cyberspace, they are increasingly viewed as a utility, like electricity in contemporary life. People are enablers, facilitators, or users in all aspects of cyberspace, but are only addressed in the current map specifically in relation to cyber leadership and the cyber workforce. Processes, including policies, resources, and management, are integral to all aspects of cyberspace, and are addressed as concepts where appropriate.

4. The first iterations of the map were messy. The earliest versions of the map contained mixed levels of abstraction and unconnected ideas. During later group mapping sessions participants recommended the design of a high level conceptual framework with fewer details to reduce the visual clutter, so details were hidden from the top view. As a repository, The Brain can capture text as well as documents, so participants suggested some standardize of formats across topics. 
5. Not all faculty members in the College have worked together on subject-related tasks, so several found this intellectual engagement worthwhile. Team mapping is better than individual mapping because it draws on multiple perspectives, challenges participants to communicate assumptions and ideas for others to understand, and fosters buy-in to the whole. Mapping provides an ideal context for negotiation of meaning, and construction of knowledge because it requires individuals to externalize their propositional frameworks (Haugwitz, Nesbit, \& Sandmann, 2011).

6. Cyberspace looks different depending upon where you sit. For example, from the perspective of cyber security, cyberspace is a vast network of machines, networks, applications and users that pose potential threats and vulnerabilities. From the perspective of cyber governance, shared values and rules of engagement evolve from communities of users who see the benefits of standards, often without authority to censure violators. The ability to understand cyberspace from various perspectives affirms its scope, complexity, immaturity, and significance to all aspects of our lives, personally and professionally, and for national security.

\section{Next Steps}

The cyberspace map is in its early stages of development. To be enhanced in value and sustained the map will need a senior-level champion. "Effective knowledge mapping is a medium of communication in which map makers speak to map users in the midst of a series of ongoing processes engaged in by map champions and map innovators "(Wexler, 2001, p. 262). Several key stakeholders of the college have viewed the evolving map and have contributed articles and ideas to enhance its content. Visitors touring the college's simulation laboratory might view the map and be invited to contribute to its evolution.

The cyberspace map can expand in its richness and value if a community of interest across organizational boundaries commits to its development using its collaborative capabilities. Likewise individual faculty members with access to the cyberspace map can add to its richness as they use it for their conference presentations, writing, curriculum development, and teaching.

Concept maps were originally designed as tools to facilitate learning. All of us are learners when it comes to cyberspace. An evolving map of this new domain and its inter-relationships, implications, potential, and side effects and benefits may help us understand cyberspace and leverage its power and potential.

\section{Disclaimer}

The views expressed in this paper are those of the authors and do not reflect the official policy or position of the National Defense University, the Department of Defense, or the U.S. Government. 


\section{References}

Ausubel, D.H. (1963). The psychology of meaningful verbal learning. New York: Grune and Stratton.

Baddeley, A. (1986). Working memory. London: Oxford University Press.

Buzan, T. (1991). Use both sides of your brain: New mind-mapping techniques (3rd ed.). New York: Plume.

Card, S., Mackinlay, J., \& Shneiderman, B. (1999). Readings in information visualization: Using vision to think. San Francisco: Morgan Kaufmann.

Davies, M. (2010). Concept mapping, mind mapping and argument mapping: What are the differences and do they matter? Higher Education, 62(3), 279-301.

Haugwitz, M., Nesbit, J. C., \& Sandmann, A. (2011). Cognitive ability and the instructional efficacy of collaborative concept mapping. Learning and Individual Differences, 20, 536-545.

Jonassen, D. H., Carr, C., \& Yueh, H. P. (1998). Computers as mindtools for engaging learners in critical thinking. TechTrends, 43(2), 24-32.

Kao, G. Y. M., \& Kuan-Chien Sun, C. (2010). Using an e-learning system with integrated concept maps to improve conceptual understanding. International Journal of Instructional Media, 37(2), 151-161.

Lamping, J., Rao, R., \& Pirolli, P. (1995). A focus+context technique based on hyperbolic geometry for visualizing large hierarchies. Proceedings of the ACM Conference Human Factors in Computing Systems, CHI, ACM. 401-408.

McLaren, T. S., Vuong, D. C. H., \& Grant, K. (2007). Do you know what you don't know? Critical reflection and concept mapping in an information systems strategy course. Communications of the Association for Information Systems, 20, 892-908.

Navarro-Prieto, R., Scaife, M., \& Rogers, Y. (1999). Cognitive strategies in web searching. Presented at the 5th Conference on Human Factors \& the Web, Gaithersburg, MD.

Novak, J. D. (1964). Importance of conceptual schemes for science teaching. Science Teacher, 31, 10-14.

Novak, J.D. (1998). Learning, creating, and using knowledge: Concept maps as facilitative tools in schools and corporations. Mahweh, NJ: Lawrence Erlbaum Associates.

Novak, J. D., \& Gowin, D. B. (1984). Learning how to learn. New York, NY: Cambridge University Press.

Ruiz-Primo, M. A., \& Shavelson, R. J. (1996). Problems and issues in the use of concept maps in science assessment. Journal of Research in Science Teaching, 33(6), 569-600.

Safayeni, F., Derbentseva, N., \& Canas, A. J. (2003). Concept maps: A theoretical note on concepts and the need for cyclic concept maps. Pensacola, FL: The Institute for Human and Machine Cognition.

Senge, P. M. (1990). The fifth discipline: The art and practice of the learning organization. New York: Currency Doubleday.

Wexler, M. N. (2001). The who, what and why of knowledge mapping. Journal of Knowledge Management, 5(3), 249-263. 


\section{Biographies}

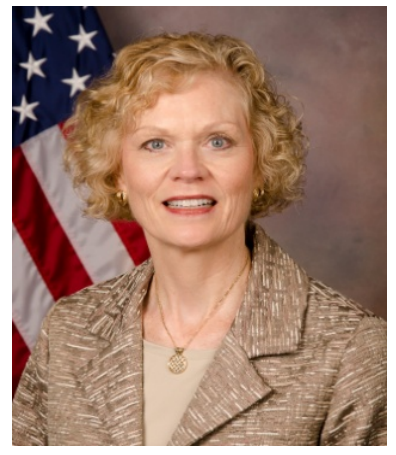

Elizabeth A. McDaniel is currently working on strategy and partnerships for the National Defense University iCollege. She served as the Dean of Faculty and Academic Programs at the Information Resources Management College, National Defense University from 1999 through 2010. After earning her Ph.D. from the University of Miami in 1978, she began her academic career at the University of Hartford, and advanced to full professor, and associate vice president for academic affairs.

Michael J. Piller is the Director of Academic Computing and leads the National Defense Univer-

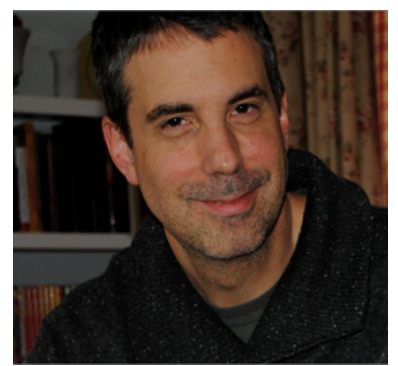
sity's iCollege Laboratory Center. The center includes two Cybersecurity Labs, a Simulation and Gaming Lab, a Crisis Management Center, an Emerging Technology Lab and Telepresence Center. He earned his Ph.D. in cognitive psychology from the Catholic University of America and has focused his career on instructional technology, human-computer interaction, the use of virtual reality/worlds technology as a learning environment, simulation and game-based learning, as well as emerging technologies and their educational applications. 